\title{
Mechanisms of resistance and susceptibility to experimental visceral leishmaniosis: BALB/C mouse versus syrian hamster model
}

Ana Nieto', Gustavo Domínguez-Bernal ${ }^{2}$, José A Orden², Ricardo De La Fuente ${ }^{2}$, Nadia Madrid-Elena ${ }^{3}$ and Javier Carrión ${ }^{2^{*}}$

\begin{abstract}
Several animal models have been established to study visceral leishmaniosis (VL), a worldwide vector-borne disease affecting humans and domestic animals that constitutes a serious public health problem. BALB/C mice and Syrian hamsters are the most widely used experimental models. In this paper, we summarize the advantages and disadvantages of these two experimental models and discuss the results obtained using these models in different studies of VL. Studies using the BALB/C mouse model have underscored differences between the liver and spleen in the course of $\mathrm{VL}$, indicating that pathological evaluation of the visceral organs is essential for understanding the immune mechanisms induced by Leishmania infantum infection. The main goal of this review is to collate the relevant literature on Leishmania pathogenesis into a sequence of events, providing a schematic view of the main components of adaptive and innate immunity in the liver and spleen after experimental infection with L. infantum or L. donovani. This review also presents several viewpoints and reflections about some controversial aspects of Leishmania research, including the choice of experimental model, route of administration, inoculum size and the relevance of pathology (intimately linked to parasite persistence): a thorough understanding of which is essential for future VL research and the successful development of efficient control strategies for Leishmania spp.
\end{abstract}

\section{Table of contents}

1. Introduction

2. Syrian hamster model of VL: suitability of this experimental model

3. Mouse model of VL: genetic control of susceptibility to L. infantum infection

4. BALB/c mouse model of VL: organ-specific immune responses

4.1. Liver: control of hepatic infection

4.1.1. Development of an immune response to the early stage of infection

4.1.2. Development of an immune response to the later stage of infection: granuloma formation

4.2. Spleen: L. infantum parasites persist and destroy the splenic architecture

\footnotetext{
* Correspondence: fjcarrion@vet.ucm.es

${ }^{2}$ Department of Animal Health, Faculty of Veterinary, Complutense University of Madrid, 28040 Madrid, Spain

Full list of author information is available at the end of the article
}

4.2.1. The acute phase of infection

4.2.2. The chronic phase of infection

4.2.3. Pathological changes in the spleen

5. Remarks and discussion

6. Competing interests

7. Authors' contributions

8. Acknowledgments

9. References

\section{Introduction}

The parasitic protozoa of the genus Leishmania cause a spectrum of diseases in humans, ranging from subclinical cutaneous infections to more serious disseminating diffuse cutaneous, mucocutaneous and visceral forms of the disease. Leishmaniosis is one of the most prevalent neglected tropical diseases affecting public health worldwide $[1,2]$. It is transmitted by the bite of female sandflies. In developing countries it is associated with extreme poverty. It is estimated that at least 20 million
C Biomed Central

(c) 2011 Nieto et al; licensee BioMed Central Ltd. This is an Open Access article distributed under the terms of the Creative Commons Attribution License (http://creativecommons.org/licenses/by/2.0), which permits unrestricted use, distribution, and reproduction in any medium, provided the original work is properly cited. 
people are infected with Leishmania. The visceral form is the most severe form of the disease. Annually, there are approximately 500000 new cases of visceral leishmaniosis (VL) [3]. Leishmania donovani is the primary cause of VL in the Indian subcontinent and East Africa, L. infantum in the areas surrounding the Mediterranean Sea where it is a zoonosis, and L. chagasi in the New World. The last two species are identical. Human beings are the only known reservoir of $L$. donovani, while canines provide the reservoir for L. infantum and L. chagasi [4]. However, since asymptomatic parasitemic injecting drug users who share injecting devices seem to be a suitable reservoir for $L$ infantum, an artificial anthro-ponotic cycle would be completed. Needles and syringes would be the vectors and uninfected injecting drug users the receptors [5]. Also, L. infantum is known to cause opportunistic infections in patients with HIV/ AIDS [6]. Canis familiaris is the major host for these parasites, and the main reservoir for human visceral infection [7]. The risk for reintroduction of VL and other vector-borne diseases in Europe as a consequence of global warming has recently been highlighted [8]. Indeed, VL appears not to be limited to the Mediterranean region and has now spread northwards [9].

Manifestations of VL can vary from asymptomatic infection to progressive fatal visceral disease. Disease progression is dependent on both the species of Leishmania involved and the genetics and immune status of the host. Active VL is characterized by fever, weight loss, hypergammaglobulinemia, hepatosplenomegaly, anemia, thrombocytopenia, leukopenia and immunodepression $[10,11]$. Also, the presence of parasite-specific antibodies forming immune complexes in the kidneys may lead to the development of glomerulonephritis $[12,13]$.

Leishmaniosis diagnosis and treatment are expensive. Despite considerable advances, there are still no efficient vaccines available against human leishmaniosis $[14,15]$. Recently, a vaccine containing the fucose-mannose ligand has been industrialized and licensed for commercialization in Brazilian endemic areas under the name of Leishmune $^{\circledR}$ (Fort Dodge Ltda, São Paulo, Brazil) to prevent canine VL. Unfortunately, the immune response induced by vaccination has not yet been fully investigated. Also, this vaccine is solely recommended for asymptomatic and seronegative dogs [16-19].

L. infantum has a digenetic life-cycle (Figure 1), alternating between free-living, flagellated, promastigotes in phlebotomine sand flies and obligate, intracellular, aflagellated amastigotes, which preferentially multiply within macrophages or dendritic cells (DCs) of the vertebrate host $[20,21]$.

Several experimental models of VL have been developed, but none of these entirely reproduce the disease in humans [22]. Much of the literature from these models documents the immune parameters contributing to resistance against the visceralizing Leishmania species used in vaccine studies [23]. This contrasts with a limited number of studies which have been prompted to study pathological aspects related to VL. In this context, close attention should be given to the histopathological alterations. Humans, dogs and hamsters often exhibit severe clinical signs and symptoms during visceral infection [23-25], whereas mice generally show a few minor signs or no clinical signs at all, depending mainly on the size of the parasite inoculum [26]. Under experimental conditions, progression of visceral disease also depends on the route of infection together with the strain of Leishmania parasites used [22]. These factors make the choice of a suitable laboratory model difficult. Studies using experimental murine models of VL do not allow exact extrapolations to be made concerning susceptibility in dogs and humans, but increase the ease of identifying genes and predicting their functional roles, as well as investigating the immune mechanisms involved in human and canine leishmaniosis. This review will aim to provide a better understanding of a variety of pathological-immune responses that have been described to date in the most widely used experimental models of VL (Syrian hamsters and BALB/c mice). Combining research approaches at the immunological, pathological and genetic levels helps to advance our understanding of the mechanisms involved in visceral infection at different stages of the disease.

\section{Syrian hamster model of VL: suitability of this experimental model}

The usual routes of infection in the hamster model of $\mathrm{VL}$ are intracardiac and intraperitoneal. However, the administration of parasites by the saphenous vein in order to minimize stress on the hamsters has also been reported [27]. Experimental studies in L. infantum and L. donovani-infected Syrian hamsters (Mesocricetus auratus) often reveal several clinical signs of progressive VL (hypergammaglobulinemia, hepatosplenomegaly, anemia, cachexia and immunodepression) that closely mimic active canine and human disease $[22,23,25,28,29]$. Surprisingly, there are significant amounts of Th1 cytokines (IFN- $\gamma$, IL-2 and TNF- $\alpha$ ) in the spleen, but there is little or no IL-4. However, to allow the parasites to multiply, deactivating Th2 cytokines (TGF- $\beta$ and IL-10) may act on infected macrophages as well as anti-Leishmania antibodies (which have no protective role in leishmaniosis) that opsonize amastigotes and induce IL-10 production in macrophages. These high activation and deactivation processes are likely to occur mainly in the spleen and liver [30]. Interestingly, Syrian hamsters exhibit reduced expression of the gene encoding inducible 


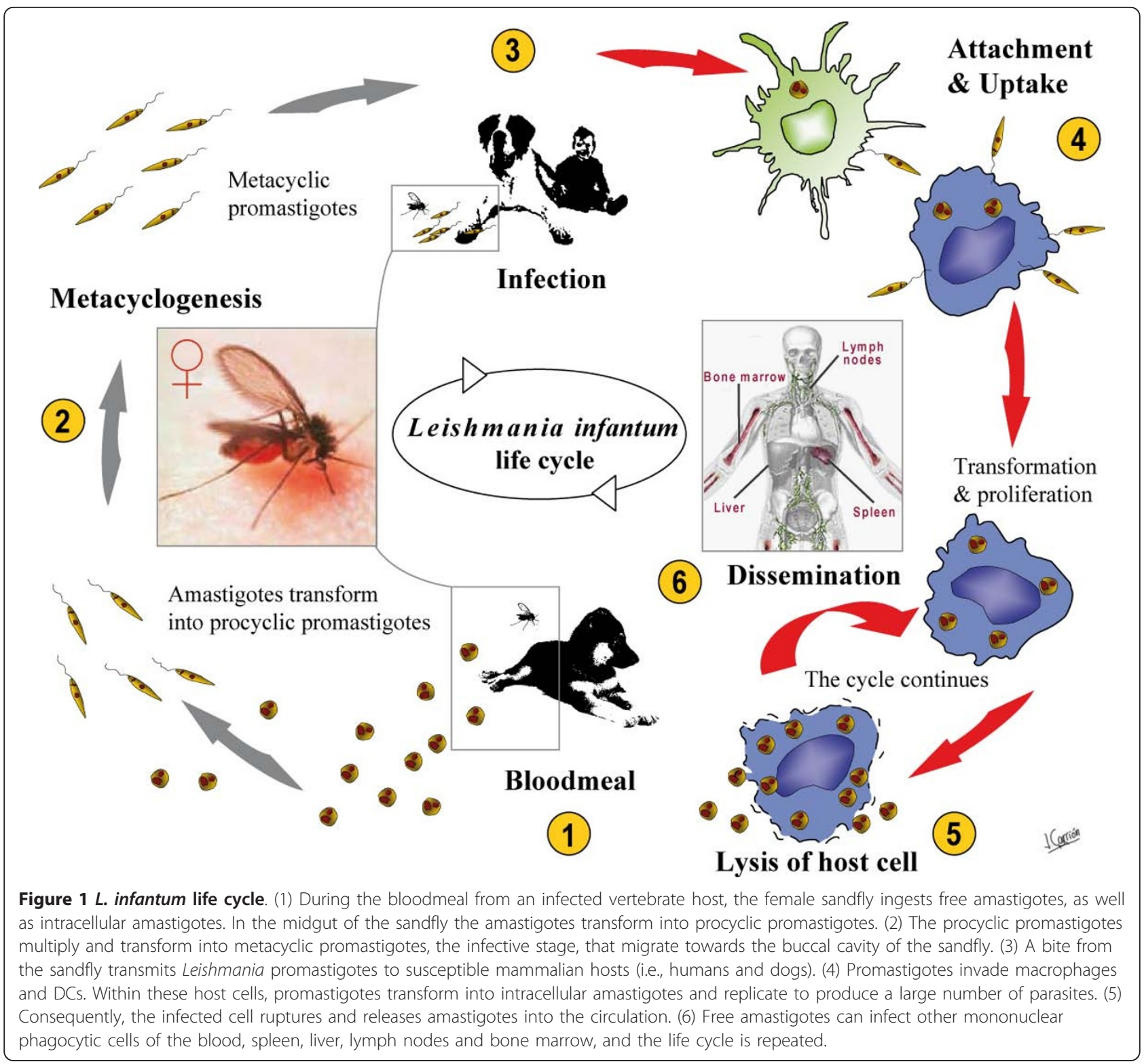

nitric oxide synthase (iNOS) in response to IFN- $\gamma$, and this is thought to lead to a low nitric oxide (NO) generation, subsequently defaulting in parasite killing $[10,28,31]$. Furthermore, there is a lack of reagents for immunological analysis in the hamster model of VL. Taking these factors into account, we consider the Syrian hamster to be a suitable experimental model for the study of the pathological features of active VL (as described below), but it is not a suitable model for the evaluation of immunization strategies, as a result of the animal's high innate susceptibility.

In Syrian hamsters, manifestations of VL can range from asymptomatic and oligosymptomatic infections to progressive fatal visceral disease [28]. The pathological features reported during VL include hypoplasia of the white pulp in the spleen, hepatic granulomas and the deposition of a secondary amyloid substance both in the spleen and the liver [32,33]. Also, other studies of active VL have reported that infected hamsters develop glomerulonephritis associated with deposition of immunoglobulins and parasite antigens (immune complexes) in the kidneys. Finally, the disseminated amyloidosis and glomerulonephritis produce renal failure and nephrotic syndrome in infected hamsters [12,34]. The visceral infection in hamsters also induces pathological alterations in hepatocytes, mainly in the endomembrane system and the peroxisomal compartment, leading to a disturbance of liver metabolism [35]. In a recent study [36], hamsters infected with L. infantum were shown to develop analogous inflammatory myopathies to those 
observed in naturally infected dogs [37]. Taken together, all these factors probably contributed to the immune response disorders that resulted in the death of the animals [22,33].

Pathological studies from our laboratory showed that after L. infantum intracardiac infection, hamsters exhibited severe histopathological alterations in both the spleen and liver at the peak of parasite burden. Among these alterations, we detected the apparition of granulomas in different maturation stages and giant cell granulomas with amastigotes in the liver (Figure 2A-D), as well as disruption of the splenic architecture accompanied by lymphoid depletion (Figure 2E-F). Interestingly, several months after intracardiac infection with $10^{7}$ promastigotes of L. infantum, we found external mucocutaneous lesions localized in the snout, accompanied by ulcers on the back of the animals (Figure 3).

\section{Mouse model of VL: genetic control of susceptibility to $L$. infantum infection}

Genetic control studies of various host defense mechanisms in the mouse (Mus musculus) model during the course of progressive infection with visceralizing Leishmania spp. are summarized in Table 1. These experiments made an important contribution in identifying genes involved in VL innate and acquired immunity. Identification of the Slc11a1 gene aided our understanding of the susceptibility at early stages of infection in $\mathrm{BALB} / \mathrm{c}$ mice, which reflects the strength of the innate immune response in controlling early parasite growth independently of acquired immune mechanisms. The Slc11a gene also controls susceptibility to bacteria. Indeed, mutations in Slc11a1 cause susceptibility to infection with Salmonella spp. [38] and Mycobacteria spp. [39]. Interestingly, iron is required for replication of pathogens such as Leishmania parasites in phagosomes. The Slc11a1 gene encodes a protein expressed on the membrane of infected phagosomes that removes $\mathrm{Fe}^{2+}$ and $\mathrm{Mn}^{2+}$ ions from the intra-phagosomal compartment restricting intracellular Leishmania multiplication in iron-limited intracellular environments [40,41]. Genetically resistant mouse strains (e.g., CBA) possess a functional Slc11a1 gene which confers innate resistance to early Leishmania parasite growth. In contrast, susceptible mice strains (e.g., C57BL/6 and BALB/c) possess a non-functional Slc11a1 gene and early parasite growth in the liver cannot be controlled [42]. However, most susceptible mouse strains, including BALB/c, develop acquired immune mechanisms to control hepatic parasite growth at later stages of infection (as previously reviewed $[43,44])$.

The parasite load in the liver at later stages of infection, which probably reflects the strength of the acquired immune response, was found to be controlled by the $\mathrm{H} 2$ and $\operatorname{Ir} 2$ loci. The haplotype at the $\mathrm{H} 2$ genomic region on chromosome 17 is involved in antigen presentation through the major histocompatibility complex (MHC). Genetic polymorphism in the MHC influences the response to numerous antigens. Several MHC haplotypes have not only been associated with resistance to leishmaniosis, but also with resistance to many other infections [45]. Differences between the $H-2^{b}$ and $H-2^{d}$ haplotypes were observed in the BALB/c background, where $\mathrm{H}-2^{b}$ resulted in lower parasite numbers in the liver than $H-2^{d}$. In addition to the liver, the $H 2$ region influences parasite numbers in the spleen and bone marrow [43]. Histopathological analysis revealed that the $\operatorname{Ir} 2$ locus in mice promoted fewer granulomas that were smaller in size, due to an efficient anti-parasite response.

The parasite burden in the spleen was also found to be controlled by the Lyst/Beige gene on chromosome 13. Indeed, homozygous $\mathrm{C} 57 \mathrm{BL} / 6 \mathrm{~J} \mathrm{bg/bg}$ (beige) mice expressed deficient natural killer (NK) cell activity and failed to eliminate L. donovani amastigotes [46].

\section{BALB/c mouse model of VL: organ-specific immune responses}

The variations in the susceptibility to VL in different strains of mice were first described nearly 40 years ago [47]. In BALB/c mice, the immune response to $L$. infantum and $L$. donovani infection can vary markedly between different organs (liver and spleen) within the same animal. In the liver, the infection can resolve with subsequent immunity to re-infection, whereas in the spleen, Leishmania parasites can persist [48]. A schematic view of the organ-specific immune responses after experimental infection with $L$. infantum in susceptible BALB/c mice is shown in Figures 4 and 6.

\subsection{Liver: control of hepatic infection \\ 4.1.1. Development of an immune response to the early stage of infection}

After being inoculated into the lateral vein of the tail, the parasites enter the liver via the portal vein and invade macrophages and DCs. In both these types of host cell, promastigotes transform into amastigotes. At this point, the innate immune system constitutes its first line of defence against Leishmania parasites. The parasitized resident macrophages (Kupffer cells, KCs) secrete chemokines (CCL3, CCL2 and CXCL10) that stimulate the recruitment of monocytes and granulocytes [44]. Despite the activation of these mechanisms in mice, amastigotes survive during the hepatic acute phase (up to two weeks post-infection (pi)) in an environment with small quantities of inflammatory cytokines, in the absence of activated $\mathrm{T}$ cells. Consequently, the number of parasites in the liver reaches a peak (Figure 4A). Nevertheless, the parasite burden may decrease 


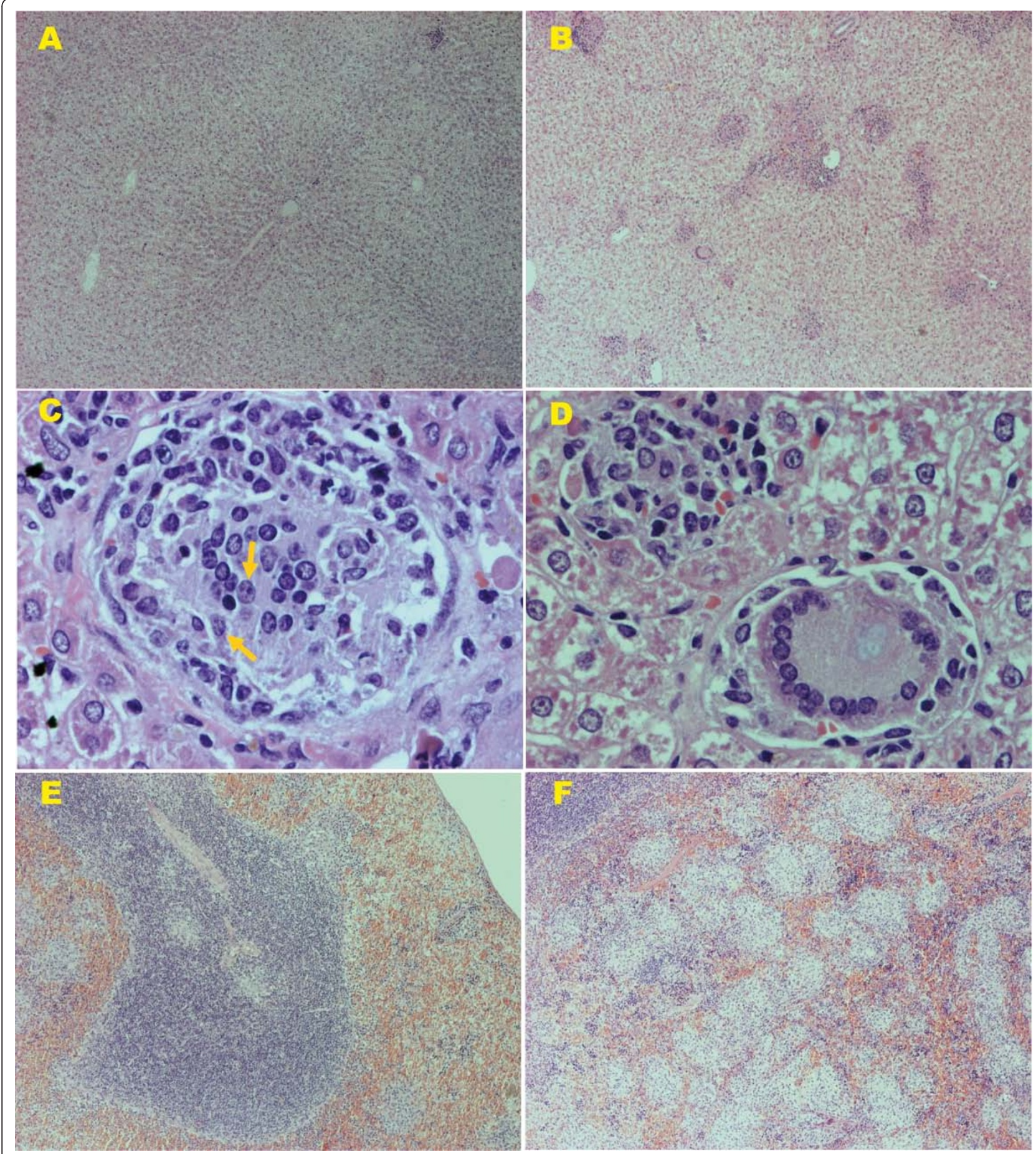

Figure 2 Liver and spleen histological sections from Syrian hamsters stained with H\&E. (A) Uninfected hamsters show normal liver histological sections ( $\times 40)$. (B) Hamsters infected with $10^{5} \mathrm{~L}$. infantum parasites show granuloma reactions after three months pi ( $\left.\times 40\right)$. Compare (A) with (B). (C) Granuloma formation. Initial parasitization of KCs (arrows) surrounded by a few inflammatory cells (lymphocytes and monocytes), showing the lack of organization after three months pi ( $\times 400)$. (D) Developing granuloma and giant cells containing few residual amastigotes after three months pi $(\times 400)$. (E) Normal splenic architecture in control hamsters $(\times 40)$. (F) Disruption of the splenic architecture accompanied with lymphoid depletion in hamsters infected with $10^{7} \mathrm{~L}$. infantum promastigotes after three months pi (X40). Compare (E) with (F). Hamsters and BALB/c mice were purchased from Harlan Interfauna Ibérica S.A. (Barcelona, Spain). The animals were maintained under conventional conditions approved by the Ethical Committee for the Animal Experimentation of the Complutense University of Madrid. 

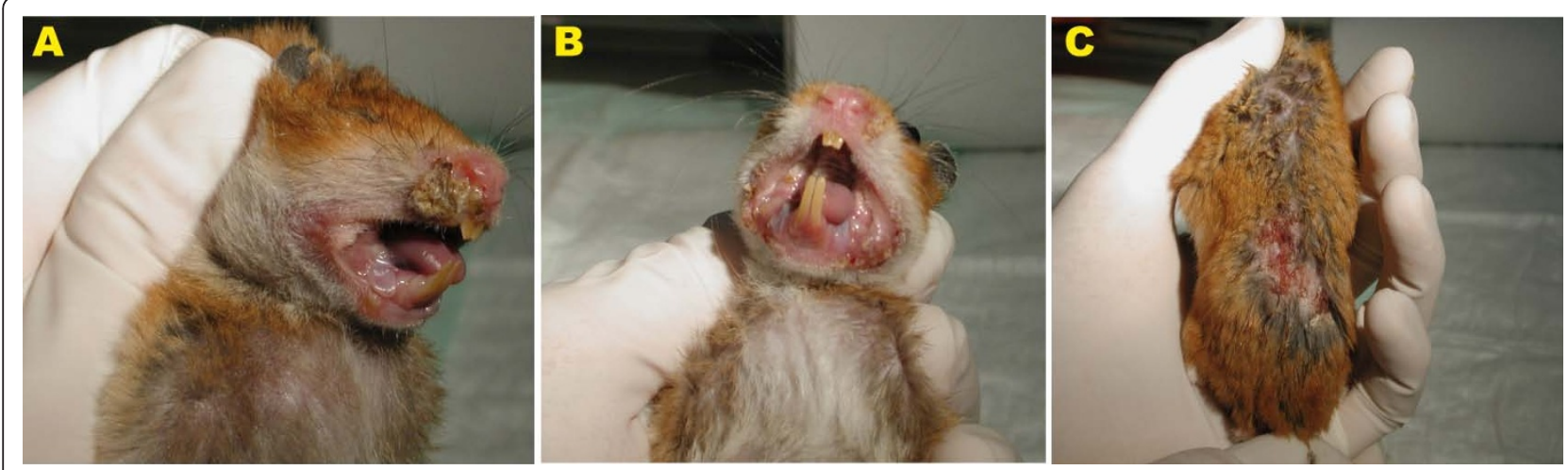

Figure 3 External lesions observed in Syrian hamsters infected with $10^{7} \mathrm{~L}$. infantum promastigotes at seven months pi. (A-B) Mucocutaneous lesions localized in the snout. (C) Ulcers on the back of the hamsters. The isolate M/CAN/ES/96/BCN150 (zymodeme MON-1) of L. infantum was used for infection experiments. This strain was maintained in our laboratory by passage in Syrian hamsters.

dramatically with the acquisition of the granulomatous response during the next stage of infection, as described below.

\subsubsection{Development of an immune response to the later stage of infection: granuloma formation}

Recent research favours a model in which Leishmaniaspecific $\mathrm{T}$ lymphocytes are pre-activated in the spleen and then migrate to the liver [48]. Once there, activated $\mathrm{T}$ cells interact with parasitized DCs, serving as a critical source of IL-12 production, that then triggers the subsequent Leishmania-specific CD $4^{+}$Th1 effector response during the later stage of infection [21]. Interestingly, activated DCs can also trigger NK cell cytotoxicity and the production of IFN- $\gamma$ [49]. In contrast to DCs, the production of IL-12 is blocked in infected macrophages. Consequently, the parasite-carrying macrophages are incompetent at priming $\mathrm{CD} 4^{+} \mathrm{T}$ cells or stimulating antigen-specific $\mathrm{CD} 4^{+} \mathrm{T}$ cells [50]. Therefore, the interaction of Leishmania-specific CD4 ${ }^{+} \mathrm{T}$ cells with infected DCs in the liver provides the proinflammatory (Th1) environment required for efficient granuloma formation (Figure 4B), which includes IL-12, IFN- $\gamma$, TNF- $\alpha$ and IL-2 production [10,48,51-53]. It is at this stage of infection (weeks 2-4 pi) that the acquired hepatic immune response is initiated. Simultaneously, the fusion of infected KCs to form multinucleated cells also contributes to inflammatory cytokine production during granuloma formation $[54,55]$. In BALB/c mice, acquired hepatic resistance to $L$. infantum clearly depends upon granuloma development. Thus, the structure of a mature tissue granuloma consists of a core of fused, parasitized $\mathrm{KCs}$ with an encircling mononuclear cell mantle containing blood monocytes and both $\mathrm{CD}^{+}$and $\mathrm{CD} 8^{+} \mathrm{T}$ cells. In some instances, B cells, plasma cells and granulocytes are also attracted. In immunologically active granulomas, antigen-presenting DCs and cytokinesecreting $\mathrm{T}$ cells are required for antimicrobial activity [54]. The formation of a granuloma is not always associated with parasite control, and the effectiveness of hepatic granulomas to kill parasites depends on their degree of maturation [52,54]. It appears that the TNF family of cytokines are not involved in the formation of granulomas but instead are involved in their maturation, as well as the maintenance of splenic architecture [42].

Granulomas become fully evolved by $2-4$ weeks pi. The overall antimicrobial efficacy of the granulomatous response appears to be variable, and only mature granulomas develop efficient leishmanicidal mechanisms to kill parasites. In contrast, developing granulomas have been reported to be less efficient at killing Leishmania parasites. Among other factors, granuloma development has been found to vary depending on the initial inoculum size. Indeed, higher numbers of mature and sterile granulomas are observed in mice infected with a

Table 1 Genes that control the immune response to $L$. donovani/L. infantum infection

\begin{tabular}{|c|c|c|c|}
\hline Host defense mechanism(s) & Locus or gene & Chromosome & Reference(s) \\
\hline Innate intraphagosomal control of infection in the spleen and the liver & Slc11a1 & 1 & {$[40-44]$} \\
\hline $\begin{array}{c}\text { Influences antigen presentation during the acquired immune response in the splen, the liver } \\
\text { and the bone marrow }\end{array}$ & $\mathrm{H} 2$ & 17 & {$[43,45]$} \\
\hline Formation of hepatic granulomas. Acquired immune response & $1 / 2$ & 2 & {$[43]$} \\
\hline $\begin{array}{c}\text { Influences resistance to parasites in the spleen. } \mathrm{C} 57 \mathrm{BL} / 6 \mathrm{~J} \mathrm{bg} / \mathrm{bg} \text { mice expressed deficient } \\
\text { natural killer cell activity and failed to eliminate } \mathrm{L} \text {. donovani amastigotes }\end{array}$ & Lyst/Beige & 13 & {$[46]$} \\
\hline
\end{tabular}




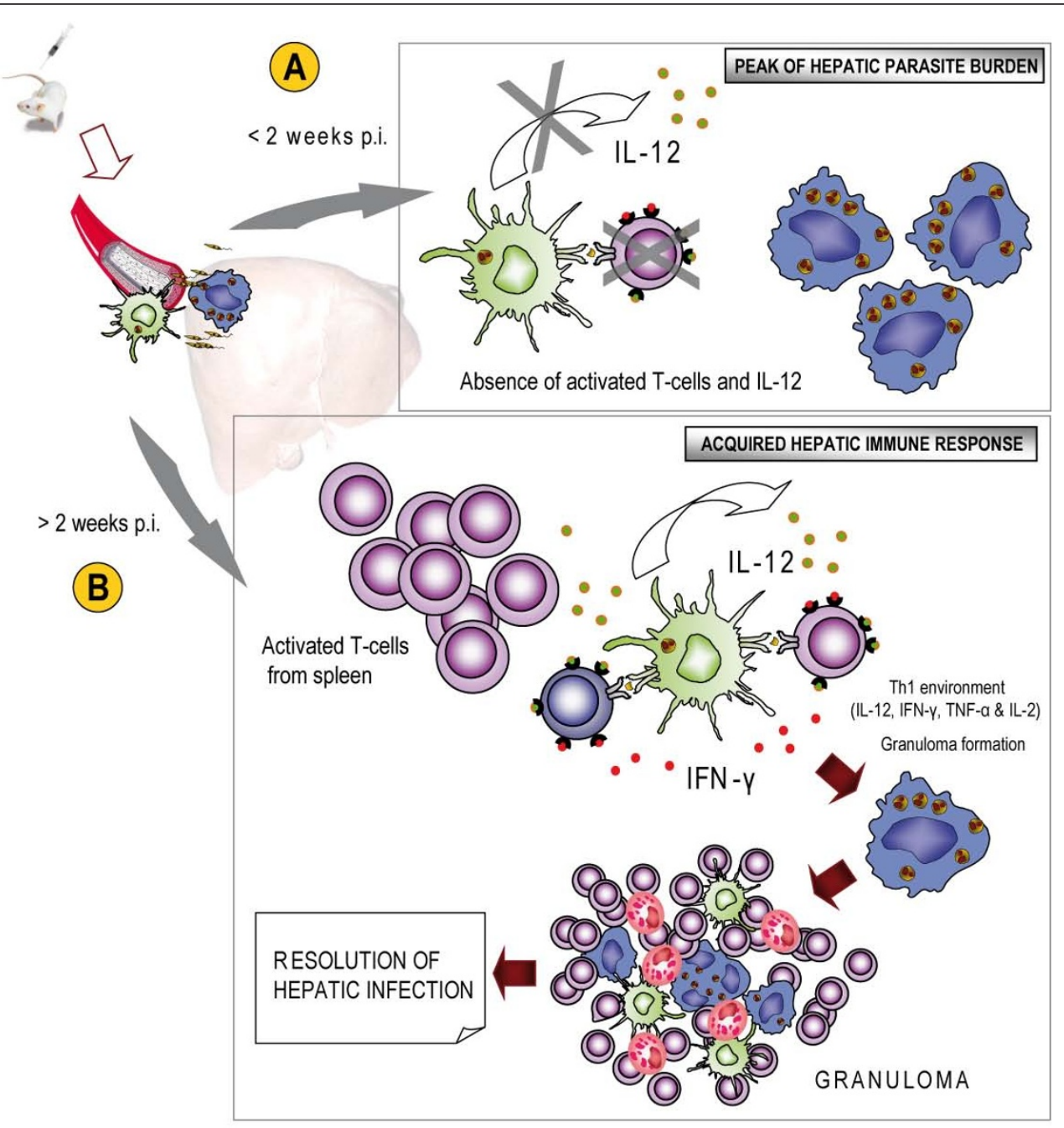

(C)

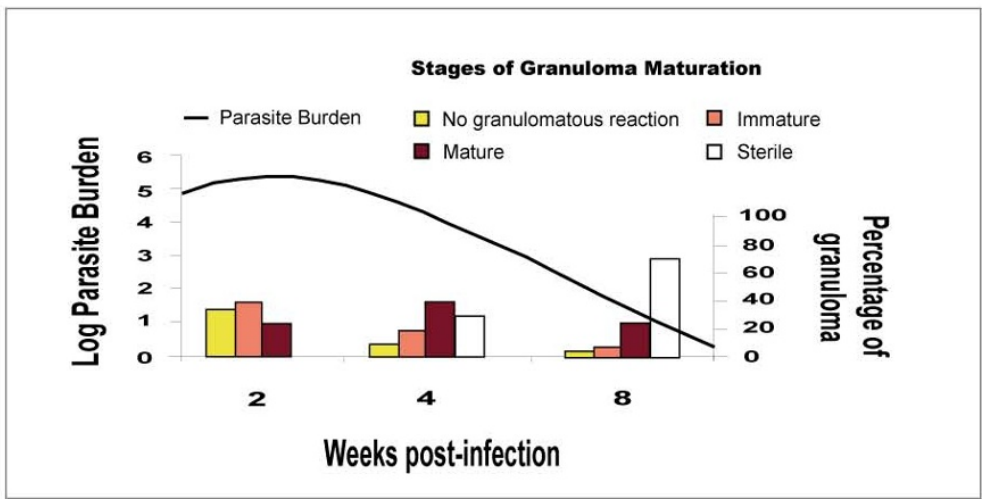

Figure $4 \mathrm{~A}$ schematic view of the immune responses in BALB/c mice livers after experimental infection with $L$. infantum. After intravenous inoculation, the parasites enter the liver and invade macrophages and DCs. (A) During the hepatic acute phase (up to two weeks pi), Leishmania amastigotes multiply in the absence of both IL-12 production and activated T-cells. Consequently, the number of parasites in the liver reaches a peak. (B) Two weeks pi, Leishmania-specific T lymphocytes migrate to the liver from the spleen and the acquired hepatic immune response is initiated. The interaction of Leishmania-specific T cells with infected KCs and DCs provides the proinflammatory (Th1) environment required for efficient granuloma formation, resulting in the resolution of hepatic infection. (C) The kinetics of parasite burden and different stages of granuloma maturation in the liver after L. infantum infection. Infected KCs with no granulomatous reaction and immature granulomas were observed in high numbers at 14 days pi but these initial stages of granuloma formation decreased in number during the course of infection, developing mature and sterile granulomas. Significantly, by 56 days pi, the number of sterile granulomas, in which the amastigotes were killed, increased and consequently, the Leishmania parasite burden decreased. Finally, the infection in the liver of BALB/c mice was resolved. 
low-inoculum size than in those infected with a highinoculum size [26]. In structurally mature hepatic granulomas, the elaboration of leishmanicidal reactive oxygen intermediates (ROIs) and reactive nitrogen intermediates (RNIs) is essential for parasite killing within infected KCs and DCs $[44,54]$.

There are various classification schemes for granulomatous inflammation in VL. Murray et al. [54] reported a summary of liver granuloma structure-function relationships in experimental VL. To score the progression of the granulomatous response, Stager et al. [56] also classified the infected focus as follows: (1) an infected $\mathrm{KC}$ with no associated cellular infiltrate, (2) an early granuloma comprising an infected $\mathrm{KC}$ surrounded by a few inflammatory cells, with no organization, (3) a mature granuloma with an organized structure, or (4) a sterile granuloma, in which amastigotes had been killed as a result of effective antileishmanial immunity. Following the above criteria our laboratory data also revealed that the resolution of disease in the livers of mice infected with L. infantum correlates with granuloma development (Figure 4C and Figure 5A). Early in the course of infection, granulomas at various stages of maturation are apparent [44]. Thus, relatively mature granulomas can be readily detected alongside infected KCs that have no associated cellular infiltrate at around four weeks pi (Figure 4C). Infected KCs exhibiting no granulomatous reaction and immature granulomas (Figure 5B) were observed in high numbers at two weeks pi, but their numbers decreased during the course of infection as mature (Figure 5C), sterile (Figure 5D) granulomas developed, in which the amastigotes were killed. After eight weeks pi, sterile granulomas gradually dissembled in an involution process [54]. Although

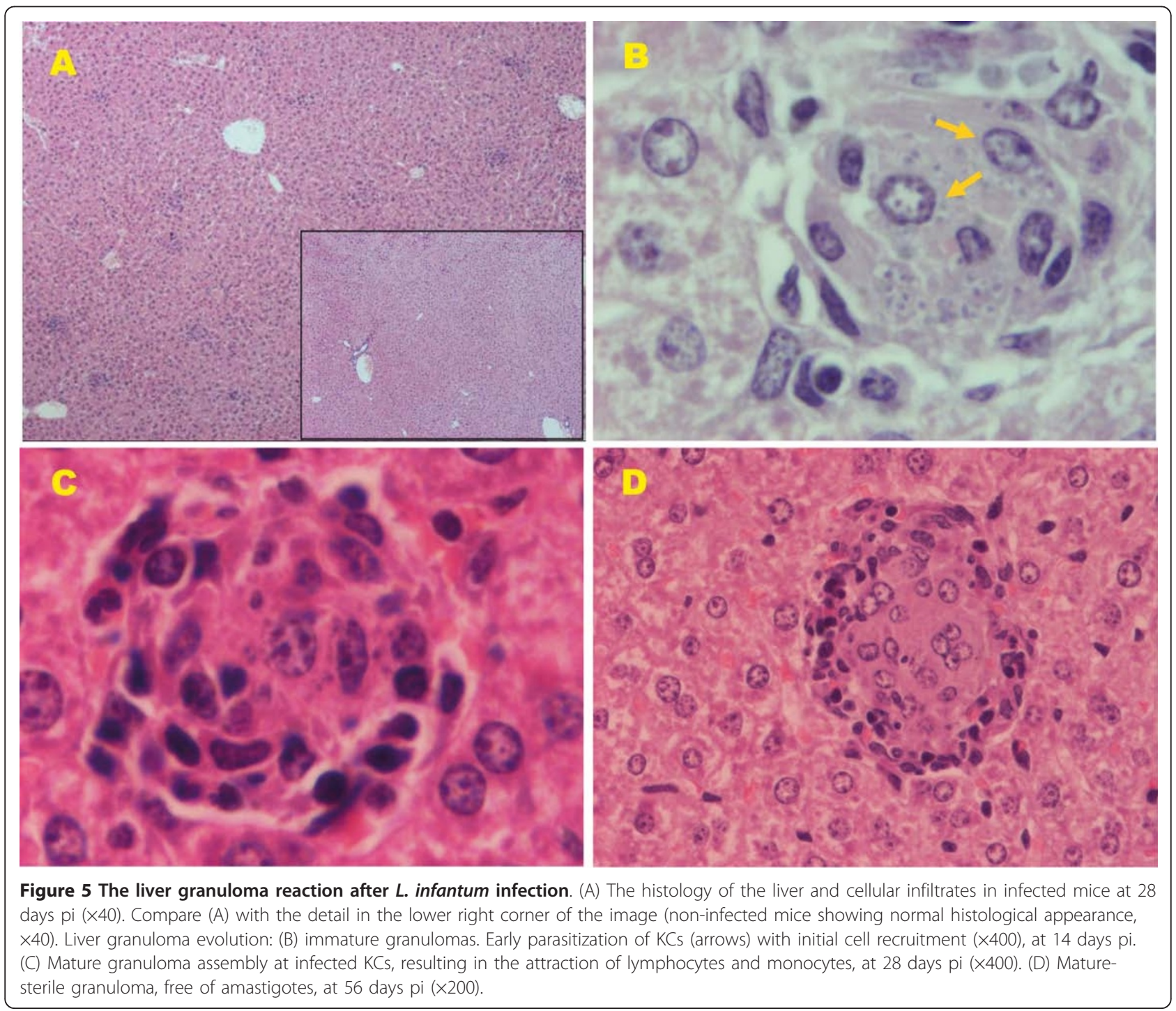


sterile cure is never achieved in the liver, parasite growth is controlled without inducing pathology and it is resistant to secondary infections with L. infantum [44]. It is possible that parasite persistence might mediate long-term immunity in the liver in a similar manner to that seen in the cutaneous leishmaniosis model caused by low-dose infection with L. major [57].

Studying the granulomatous response is important because granuloma development has been associated with Leishmania infection in the liver, as demonstrated in rodent models. Moreover, enhanced granuloma maturation represents a good marker of successful vaccination against VL [58].

\subsection{Spleen: visceralizing Leishmania parasites persist and destroy the splenic architecture}

In contrast to the liver, the spleen and bone marrow become chronically infected in mice [44]. The immune response to L. infantum in the spleen (Figure 6A-C) can be separated into two phases: acute and chronic.

\subsubsection{The acute phase of infection}

Following intravenous experimental infection in mice, $L$. infantum promastigotes enter the spleen via the splenic artery and are rapidly removed from the circulation in the spleen by marginal zone (MZ) macrophages and rarely by DCs. It is likely that the majority of DCs acquire Leishmania antigens by phagocytosis of infected macrophages or their remnants in the MZ $[44,59]$. Within these cells, promastigotes replicate intracellularly as amastigotes. In the spleen, MZ macrophages phagocytose $>95 \%$ of intravenously administered L. infantum parasites, where $>50 \%$ of the initial parasite inoculum is killed within $24 \mathrm{~h}$ of infection [48]. It appears that DCs acquire parasite antigens within the MZ (Figure 6A) and subsequently migrate to the periarteriolar lymphoid sheath (PALS). Once in the PALS, DCs secrete IL-12 [60] and present parasite antigens to $\mathrm{T}$ and $\mathrm{NK}$ cells, resulting in the activation of these effector cells (Figure 6B). Interestingly, L. infantum infection stimulates IL-12 production by splenic DCs within the PALS, but not infected macrophages within the MZ [61]. As described above, evidence suggests that Leishmaniaspecific $\mathrm{T}$ lymphocytes are primed in the spleen during the acute stage of infection $(<4$ weeks) and then migrate to the liver to initiate a granulomatous response $[42,44,48,62]$.

\subsubsection{The chronic phase of infection}

During the chronic stage of infection (> 4 weeks) in the spleen, failure to resolve L. infantum infection occurs (Figure 6C) and the splenic architecture breaks down. There are at least three possible explanations for the failure of the specific effector response [48]: (1) assuming that the priming of $\mathrm{T}$ and NK cells by DCs occurs at the PALS, a site that is anatomically segregated from the $M Z$, infected macrophages fail to produce chemoattractants to bring effector cells into their vicinity. (2) Infected macrophages are unable to activate intrinsic leishmanicidal mechanisms following exposure to cytokines and ligands from $\mathrm{T}$ and NK cells. It has been reported that $L$. infantum-infected macrophages fail to produce IL-12 and also have a reduced capacity to generate both ROIs and NO, which are important microbicidal molecules for killing intracellular pathogens $[44,54,61]$. (3) Failure in the development of the efficient granulomatous immune effector response occurs in the spleen. Together, the low expression levels of MHC class II on L. infantum macrophages and their intrinsic defects in the generation of an antileishmanial response (see above), contribute to failure to form inflammatory foci around infected MZ macrophages [48].

Any of these three possibilities may contribute to the failure of the spleen to resolve murine VL. Paradoxically, the spleen is an initial site for the generation of cellmediated immune responses, but ultimately becomes a site of parasite persistence, with associated immunopathological changes [44].

\subsubsection{Pathological changes in the spleen}

In the spleen, $L$. infantum parasite persistence is accompanied by a failure of granuloma formation, splenomegaly and other pathological changes, such as the disruption of splenic microarchitecture, including the disintegration of the white pulp accompanied by the destruction of follicular DCs, and the absence of germinal centres $[10,22,24,42,48,61,63]$. Interestingly, there is evidence that high levels of TNF mediate the destruction of $\mathrm{MZ}$ macrophages, while IL-10 promotes impaired DC migration into $\mathrm{T}$-cell areas with subsequent ineffective $\mathrm{T}$-cell priming [44]. Data from our laboratory showed that during the acute stage of infection ( $<4$ weeks), parasite burden and the level of splenic disruption increases with time (Figure 6D). Previously, we have reported that the intensity of lymphoid depletion can vary depending on the initial inoculum size. Indeed, higher numbers of lymphoid-depleted BALB/c mice were observed when a high-inoculum size was used compared with a low-inoculum size [26]. In agreement with previous studies [44], our findings revealed the progressive development of splenic pathology in mice infected with L. infantum, including disruption of tissue anatomy accompanied by the loss of germinal centers (Figure 7).

\section{Remarks and discussion}

The experimental murine model of L. infantum infection mimics many of the features of canine and human infections. Syrian hamsters also exhibit severe clinical signs and symptoms that are similar to those observed in naturally infected dogs and humans [23,24,36]. However, the absence of iNOS expression $[10,28,31]$ and the 


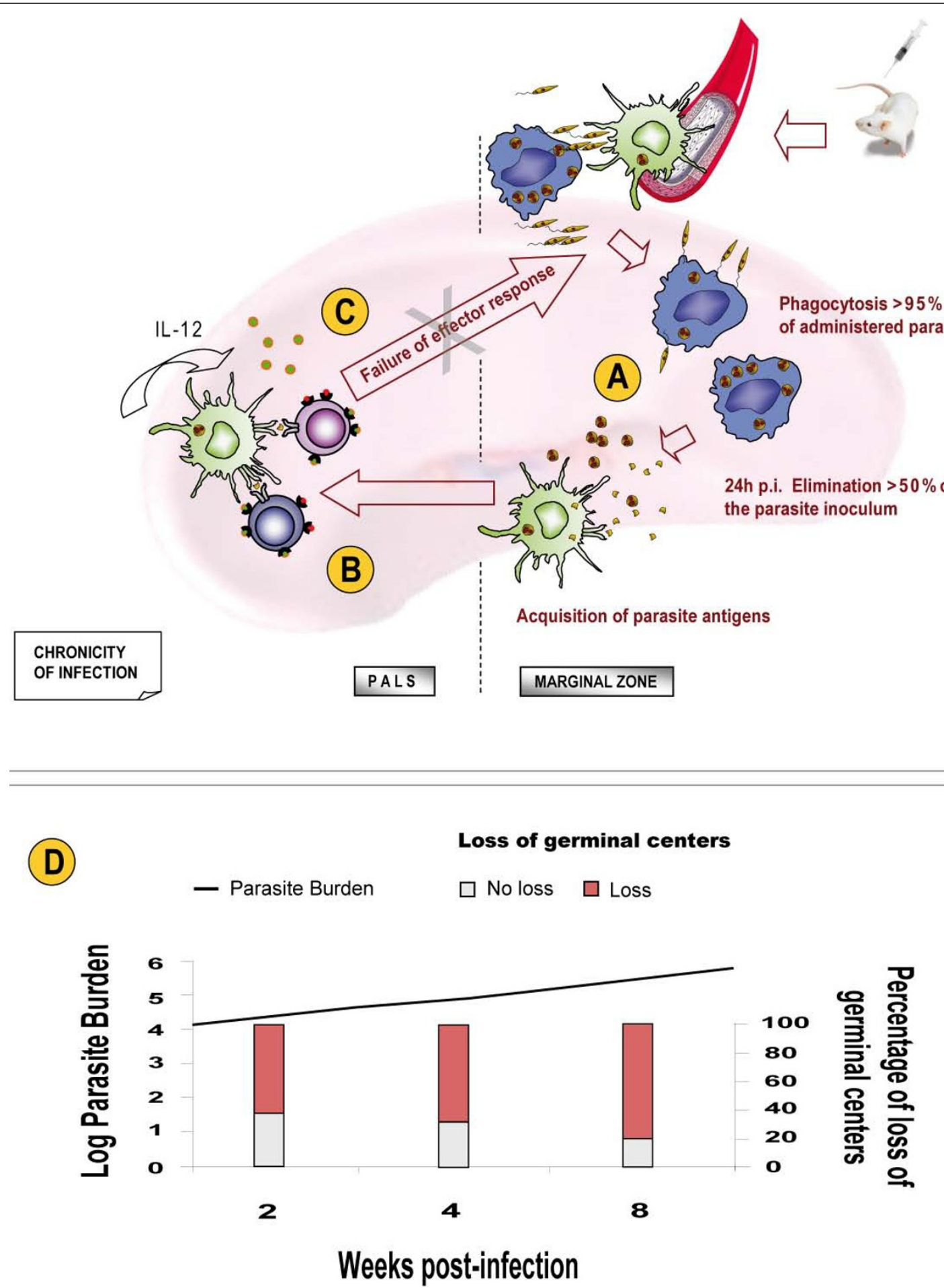

Figure $6 \mathrm{~A}$ schematic view of the immune responses in the BALB/c mice spleens after experimental infection with $L$. infantum. (A) In the initial stage of infection, parasites from the blood invade macrophages and DCs into the splenic MZ. Also, DCs acquire parasite antigens in the MZ and subsequently migrate to the PALS. (B) DCs produce IL-12 and present parasite antigens to T cells in the PALS. (C) Leishmania-specific T-cells are activated in the PALS but a failure in the specific effector response prevents them from interacting with parasitized host cells in the MZ. Finally, chronicity of infection occurs in the spleen. (D) The kinetics of parasite burden and loss of germinal centers in the spleen after L. infantum infection. The progressive loss of splenic germinal centers increased with time. Thus, in the spleen, a site of chronic infection, the high levels of depletion in the white pulp at 56 days pi correlated with the high Leishmania parasite burden. 


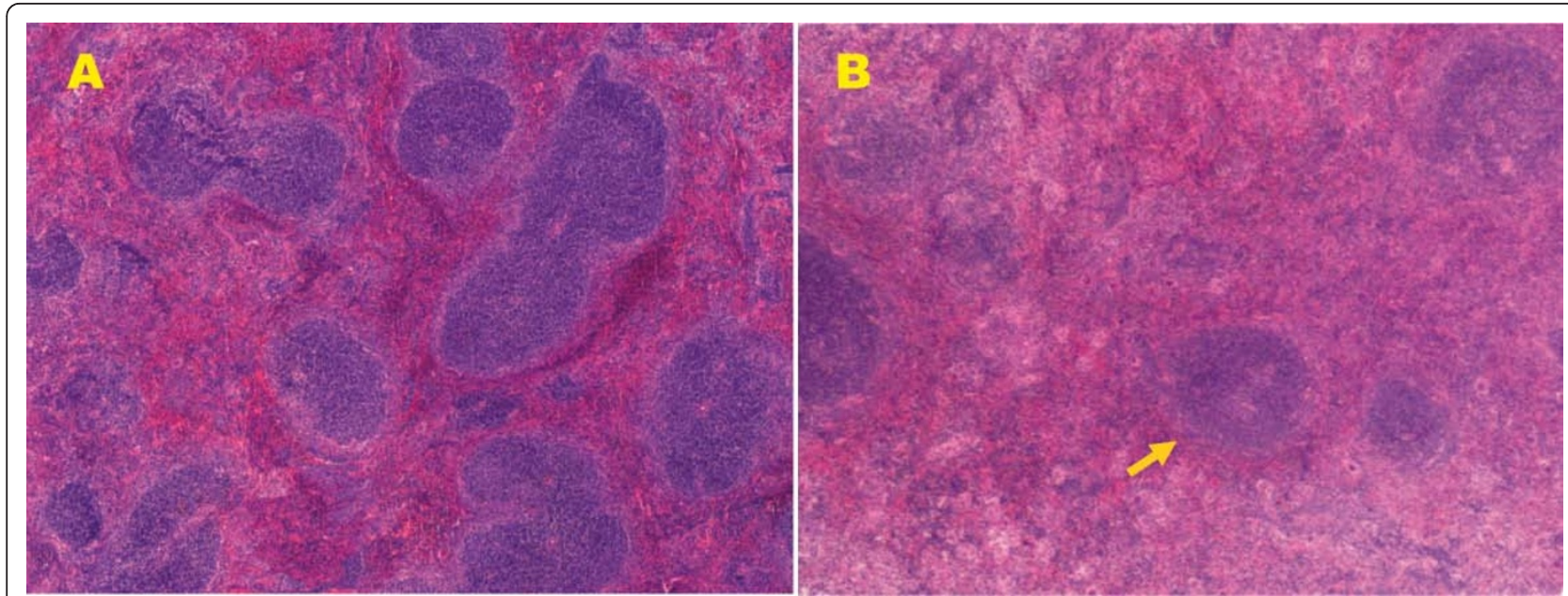

Figure 7 Changes in splenic anatomy after $L$. infantum infection. (A) Non-infected mice showed normal histological appearance $(\times 40)$. (B) After 56 days pi, the splenic architecture showed a significant loss of germinal centers (arrow) in the white pulp ( $\times 40)$. Compare (A) with (B).

suppression of lymphoproliferative responses $[64,65]$ observed, lead us to argue that the hamster is a more suitable model for pathological studies of VL than for the evaluation of vaccine candidates. However, to date, most researchers have elected to use the BALB/c mouse model for investigating disease pathogenesis of $\mathrm{VL}$, as well as for vaccine studies $[26,44,54,66-68]$. In mice, the susceptibility to visceralizing Leishmania species is mainly determined by the Slc11a1 gene that encodes a phagosomal component that confers the ability to control the early infection (as described above). Even so, $\mathrm{BALB} / \mathrm{c}$ mice, lacking this gene, are able to control the infection at a later stage $[42,69]$. In this context, BALB/C mice provide a better model of self-healing or subclinical infection than of disseminated visceral disease [10]. Paciello et al. [36] reported that susceptible mouse strains do not reproduce progressive disease as observed in human active VL. Furthermore, the intensity of pathological changes in the visceral organs of BALB/c mice can vary depending on the initial inoculum size, as described above. Indeed, we proposed that infecting mice with a large inoculum constitutes a suitable model for the study of the pathological changes of VL [26].

Infection with L. infantum, either intravenously or intradermally, leads to organ-specific immune responses that are important determinants of disease outcome in $\mathrm{BALB} / \mathrm{c}$ mice $[10,48]$. Apparently, the intravenous route of inoculation does not mimic natural infection by the sandfly [22]. However, during natural infection, the blood-sucking action of the vector on the skin of the host may result in both intravenous and intradermal administration of the parasite [26]. Subsequently, parasites multiply rapidly for the first few weeks in the liver. Curiously, the spleen is the initial site of generation of specific $T$ effector cells with the ability to move to the liver. Once in the liver, the development of cell-mediated immune responses is essential for the clearance of L. infantum parasites. In contrast, the spleen ultimately becomes the site of parasite persistence $[26,44,66,70]$, suggesting that the spleen is more susceptible to L. infantum infection than the liver [71]. Interestingly, the leishmanicidal efficacy of hepatic granulomas is dependent on their degree of maturation $[26,51,56]$. Therefore, determining the degree of maturation of hepatic granulomas constitutes an effective tool for selecting VL vaccine candidates and for monitoring disease progression.

In summary, it is reasonable to suppose that understanding the development of acquired parasite-specific immunity in the liver and the reasons for effector splenic response failure in VL, may lead to the development of effective strategies for parasite clearance in host target organs during VL and new treatments for canine and human leishmaniosis.

\section{Acknowledgements}

This research was support in part by a grant from the Spanish Ministry of Education and Science (MEC) (AGL2007-62207). Francisco Javier Carrión Herrero is an investigator of the "Juan de la Cierva" program (JCl-200904069) from the Spanish Ministry of Science and Innovation (MICINN).

\section{Author details}

${ }^{1}$ Anapath, Anatomic Pathology Laboratory, 18015 Granada, Spain.

${ }^{2}$ Department of Animal Health, Faculty of Veterinary, Complutense University of Madrid, 28040 Madrid, Spain. ${ }^{3}$ Department of Infectious Diseases, Hospital Ramón y Cajal, 28034 Madrid, Spain.

\section{Authors' contributions}

AN performed the histopathological analyses and participated in the design of the study. GDB, JAO, RDF and NME participated in the design and the discussion section of this paper. JC conceived of the study, carried out the experiments and wrote the paper. All authors read and approved the final manuscript. 


\section{Competing interests}

The authors declare that they have no competing interests.

Received: 27 September 2010 Accepted: 23 February 2011

Published: 23 February 2011

\section{References}

1. Murray HW, Berman JD, Davies CR, Saravia NG: Advances in leishmaniasis. Lancet 2005, 366:1561-1577.

2. Hotez PJ, Molyneux DH, Fenwick A, Kumaresan J, Sachs SE, Sachs JD, Savioli L: Control of neglected tropical diseases. N Engl J Med 2007, 357:1018-1027.

3. Hotez PJ: Nuclear weapons and neglected diseases: the "ten-thousandto-one gap". PLoS Negl Trop Dis 2010, 4:e680.

4. Guerin PJ, Olliaro P, Sundar S, Boelaert M, Croft SL, Desjeux P, Wasunna MK, Bryceson AD: Visceral leishmaniasis: current status of control, diagnosis, and treatment, and a proposed research and development agenda. Lancet Infect Dis 2002, 2:494-501.

5. Pineda JA, Martin-Sanchez J, Macias J, Morillas F: Leishmania spp infection in injecting drug users. Lancet 2002, 360:950-951.

6. Dujardin JC, Campino L, Canavate C, Dedet JP, Gradoni L, Soteriadou K, Mazeris A, Ozbel Y, Boelaert M: Spread of vector-borne diseases and neglect of Leishmaniasis, Europe. Emerg Infect Dis 2008, 14:1013-1018.

7. Maia C, Nunes M, Cristovao J, Campino L: Experimental canine leishmaniasis: Clinical, parasitological and serological follow-up. Acta Trop 2010, 116:193-199.

8. Maroli M, Rossi L, Baldelli R, Capelli G, Ferroglio E, Genchi C, Gramiccia M, Mortarino M, Pietrobelli M, Gradoni L: The northward spread of leishmaniasis in Italy: evidence from retrospective and ongoing studies on the canine reservoir and phlebotomine vectors. Trop Med Int Health 2008, 13:256-264.

9. Bogdan C, Schonian G, Banuls AL, Hide M, Pratlong F, Lorenz E, Rollinghoff M, Mertens R: Visceral leishmaniasis in a German child who had never entered a known endemic area: case report and review of the literature. Clin Infect Dis 2001, 32:302-306.

10. Wilson ME, Jeronimo SM, Pearson RD: Immunopathogenesis of infection with the visceralizing Leishmania species. Microb Pathog 2005, 38:147-160

11. Pearson RD, Sousa AQ: Clinical spectrum of Leishmaniasis. Clin Infect Dis 1996, 22:1-13.

12. Sartori A, De Oliveira AV, Roque-Barreira MC, Rossi MA, Campos-Neto A: Immune complex glomerulonephritis in experimental kala-azar. Parasite Immunol 1987, 9:93-103.

13. Sanyal T, Ghosh DK, Sarkar D: Identification of immune complex antigens in sera of Indian kala-azar patients. Indian J Exp Biol 1991, 29:411-415.

14. Croft SL, Vivas L, Brooker S: Recent advances in research and control of malaria, leishmaniasis, trypanosomiasis and schistosomiasis. East Mediterr Health J 2003, 9:518-533.

15. Choi CM, Lerner EA: Leishmaniasis as an emerging infection. J Investig Dermatol Symp Proc 2001, 6:175-182.

16. Saraiva EM, de Figueiredo Barbosa A, Santos FN, Borja-Cabrera GP, Nico D, Souza LO, de Oliveira Mendes-Aguiar C, de Souza EP, Fampa P, Parra LE, Menz I, Dias JG Jr, de Oliveira SM, Palatnik-de-Sousa CB: The FML-vaccine (Leishmune) against canine visceral leishmaniasis: a transmission blocking vaccine. Vaccine 2006, 24:2423-2431.

17. Parra LE, Borja-Cabrera GP, Santos FN, Souza LO, Palatnik-de-Sousa CB, Menz I: Safety trial using the Leishmune vaccine against canine visceral leishmaniasis in Brazil. Vaccine 2007, 25:2180-2186.

18. Dantas-Torres F: Leishmune vaccine: the newest tool for prevention and control of canine visceral leishmaniosis and its potential as a transmission-blocking vaccine. Vet Parasitol 2006, 141:1-8.

19. de Lima VM, Ikeda FA, Rossi CN, Feitosa MM, Vasconcelos RO, Nunes CM, Goto H: Diminished CD4+/CD25+ T cell and increased IFN-gamma levels occur in dogs vaccinated with Leishmune in an endemic area for visceral leishmaniasis. Vet Immunol Immunopathol 2010, 135:296-302.

20. Alexander J, Satoskar AR, Russell DG: Leishmania species: models of intracellular parasitism. J Cell Sci 1999, 112(Pt 18):2993-3002.

21. Antoine JC, Prina E, Courret N, Lang T: Leishmania spp.: on the interactions they establish with antigen-presenting cells of their mammalian hosts. Adv Parasitol 2004, 58:1-68.

22. Handman E: Leishmaniasis: current status of vaccine development. Clin Microbiol Rev 2001, 14:229-243.
23. Hommel M, Jaffe $\mathrm{CL}$, Travi B, Milon G: Experimental models for leishmaniasis and for testing anti-leishmanial vaccines. Ann Trop Med Parasitol 1995, 89(Suppl 1):55-73.

24. Melby PC, Tabares A, Restrepo BI, Cardona AE, McGuff HS, Teale JM: Leishmania donovani: evolution and architecture of the splenic cellular immune response related to control of infection. Exp Parasitol 2001, 99:17-25.

25. Requena JM, Soto M, Doria MD, Alonso C: Immune and clinical parameters associated with Leishmania infantum infection in the golden hamster model. Vet Immunol Immunopathol 2000, 76:269-281.

26. Carrion J, Nieto A, Iborra S, Iniesta V, Soto M, Folgueira C, Abanades DR, Requena JM, Alonso C: Immunohistological features of visceral leishmaniasis in BALB/c mice. Parasite Immunol 2006, 28:173-183.

27. Lei SM, Ramer-Tait AE, Dahlin-Laborde RR, Mullin K, Beetham JK: Reduced hamster usage and stress in propagating Leishmania chagasi promastigotes using cryopreservation and saphenous vein inoculation. J Parasitol 2010, 96:103-108.

28. Melby PC, Chandrasekar B, Zhao W, Coe JE: The hamster as a model of human visceral leishmaniasis: progressive disease and impaired generation of nitric oxide in the face of a prominent Th1-like cytokine response. J Immunol 2001, 166:1912-1920.

29. Dea-Ayuela MA, Rama-Iniguez S, Alunda JM, Bolas-Fernandez F: Setting new immunobiological parameters in the hamster model of visceral leishmaniasis for in vivo testing of antileishmanial compounds. Vet Res Commun 2007, 31:703-717.

30. Goto H, Prianti MG: Immunoactivation and immunopathogeny during active visceral leishmaniasis. Rev Inst Med Trop Sao Paulo 2009, 51:241-246.

31. Goto H, Lindoso JA: Immunity and immunosuppression in experimental visceral leishmaniasis. Braz J Med Biol Res 2004, 37:615-623.

32. Wilson ME, Innes DJ, Sousa AD, Pearson RD: Early histopathology of experimental infection with Leishmania donovani in hamsters. J Parasitol 1987, 73:55-63.

33. Rica-Capela MJ, Cortes S, Leandro C, Peleteiro MC, Santos-Gomes G, Campino L: Immunological and histopathological studies in a rodent model infected with Leishmania infantum promastigotes or amastigotes. Parasitol Res 2003, 89:163-169.

34. Sartori A, Roque-Barreira MC, Coe J, Campos-Neto A: Immune complex glomerulonephritis in experimental kala-azar. II: Detection and characterization of parasite antigens and antibodies eluted from kidneys of Leishmania donovani-infected hamsters. Clin Exp Immunol 1992, 87:386-392.

35. Vianna VL, Takiya CM, de Brito-Gitirana L: Histopathologic analysis of hamster hepatocytes submitted to experimental infection with Leishmania donovani. Parasitol Res 2002, 88:829-836.

36. Paciello O, Wojcik S, Gradoni L, Oliva G, Trapani F, lovane V, Politano L, Papparella S: Syrian hamster infected with Leishmania infantum: a new experimental model for inflammatory myopathies. Muscle Nerve 2010, 41:355-361.

37. Paciello O, Oliva G, Gradoni L, Manna L, Manzillo VF, Wojcik S, Trapani F, Papparella S: Canine inflammatory myopathy associated with Leishmania Infantum infection. Neuromuscul Disord 2009, 19:124-130.

38. Nairz M, Fritsche G, Crouch ML, Barton HC, Fang FC, Weiss G: Slc11a1 limits intracellular growth of Salmonella enterica sv. Typhimurium by promoting macrophage immune effector functions and impairing bacterial iron acquisition. Cell Microbiol 2009, 11:1365-1381.

39. Roupie V, Rosseels V, Piersoel V, Zinniel DK, Barletta RG, Huygen K: Genetic resistance of mice to Mycobacterium paratuberculosis is influenced by Slc11a1 at the early but not at the late stage of infection. Infect Immun 2008, 76:2099-2105.

40. Marquis JF, Gros P: Intracellular Leishmania: your iron or mine? Trends Microbiol 2007, 15:93-95.

41. Huynh C, Andrews NW: Iron acquisition within host cells and the pathogenicity of Leishmania. Cell Microbiol 2008, 10:293-300.

42. Kaye PM, Svensson M, Ato M, Maroof A, Polley R, Stager S, Zubairi S, Engwerda CR: The immunopathology of experimental visceral leishmaniasis. Immunol Rev 2004, 201:239-253.

43. Lipoldova M, Demant P: Genetic susceptibility to infectious disease: lessons from mouse models of leishmaniasis. Nat Rev Genet 2006, 7:294-305

44. Stanley AC, Engwerda CR: Balancing immunity and pathology in visceral leishmaniasis. Immunol Cell Biol 2007, 85:138-147. 
45. Wegner KM, Kalbe M, Schaschl H, Reusch TB: Parasites and individual major histocompatibility complex diversity-an optimal choice? Microbes Infect 2004, 6:1110-1116.

46. Kirkpatrick CE, Farrell JP, Warner JF, Denner G: Participation of natural killer cells in the recovery of mice from visceral leishmaniasis. Cell Immunol 1985, 92:163-171.

47. Bradley DJ, Kirkley J: Variation in susceptibility of mouse strains to Leishmania donovani infection. Trans R Soc Trop Med Hyg 1972, 66:527-528.

48. Engwerda CR, Kaye PM: Organ-specific immune responses associated with infectious disease. Immunol Today 2000, 21:73-78.

49. Schleicher U, Liese J, Knippertz I, Kurzmann C, Hesse A, Heit A, Fischer JA, Weiss S, Kalinke U, Kunz S, Bogdan C: NK cell activation in visceral leishmaniasis requires TLR9, myeloid DCs, and IL-12, but is independent of plasmacytoid DCs. J Exp Med 2007, 204:893-906.

50. Soong L: Modulation of dendritic cell function by Leishmania parasites. J Immunol 2008, 180:4355-4360.

51. Murray HW, Squires KE, Miralles CD, Stoeckle MY, Granger AM, GranelliPiperno A, Bogdan C: Acquired resistance and granuloma formation in experimental visceral leishmaniasis. Differential T cell and lymphokine roles in initial versus established immunity. J Immunol 1992, 148:1858-1863.

52. Murray HW, Nathan CF: Macrophage microbicidal mechanisms in vivo: reactive nitrogen versus oxygen intermediates in the killing of intracellular visceral Leishmania donovani. J Exp Med 1999, 189:741-746.

53. Squires KE, Schreiber RD, McElrath MJ, Rubin BY, Anderson SL, Murray HW: Experimental visceral leishmaniasis: role of endogenous IFN-gamma in host defense and tissue granulomatous response. J Immunol 1989, 143:4244-4249.

54. Murray HW: Tissue granuloma structure-function in experimental visceral leishmaniasis. Int J Exp Pathol 2001, 82:249-267.

55. Hernandez-Pando R, Bornstein QL, Aguilar Leon D, Orozco EH, Madrigal VK, Martinez Cordero E: Inflammatory cytokine production by immunological and foreign body multinucleated giant cells. Immunology 2000, 100:352-358

56. Stager S, Alexander J, Carter KC, Brombacher F, Kaye PM: Both interleukin-4 (IL-4) and IL-4 receptor alpha signaling contribute to the development of hepatic granulomas with optimal antileishmanial activity. Infect Immun 2003, 71:4804-4807.

57. Belkaid Y, Piccirillo CA, Mendez S, Shevach EM, Sacks DL: CD4+CD25+ regulatory $T$ cells control Leishmania major persistence and immunity. Nature 2002, 420:502-507.

58. Carter KC, Henriquez FL, Campbell SA, Roberts CW, Nok A, Mullen AB, McFarlane E: DNA vaccination against the parasite enzyme gammaglutamylcysteine synthetase confers protection against Leishmania donovani infection. Vaccine 2007, 25:4502-4509.

59. Mebius RE, Kraal G: Structure and function of the spleen. Nat Rev Immunol 2005, 5:606-616

60. Ato M, Maroof A, Zubairi S, Nakano H, Kakiuchi T, Kaye PM: Loss of dendritic cell migration and impaired resistance to Leishmania donovani infection in mice deficient in CCL19 and CCL21. J Immuno/ 2006, 176:5486-5493.

61. Gorak PM, Engwerda CR, Kaye PM: Dendritic cells, but not macrophages, produce IL-12 immediately following Leishmania donovani infection. Eur J Immunol 1998, 28:687-695.

62. Engwerda CR, Ato M, Kaye PM: Macrophages, pathology and parasite persistence in experimental visceral leishmaniasis. Trends Parasitol 2004, 20:524-530.

63. Smelt SC, Engwerda CR, McCrossen M, Kaye PM: Destruction of follicular dendritic cells during chronic visceral leishmaniasis. J Immunol 1997, 158:3813-3821.

64. Dasgupta S, Mookerjee A, Chowdhury SK, Ghose AC: Immunosuppression in hamsters with progressive visceral leishmaniasis: an evaluation of the role of nitric oxide toward impairment of the lymphoproliferative response. Parasitol Res 1999, 85:594-596.

65. Mookerjee A, Sen PC, Ghose AC: Immunosuppression in hamsters with progressive visceral leishmaniasis is associated with an impairment of protein kinase $C$ activity in their lymphocytes that can be partially reversed by okadaic acid or anti-transforming growth factor beta antibody. Infect Immun 2003, 71:2439-2446.
66. Ahmed S, Colmenares M, Soong L, Goldsmith-Pestana K, Munstermann L, Molina R, McMahon-Pratt D: Intradermal infection model for pathogenesis and vaccine studies of murine visceral leishmaniasis. Infect Immun 2003, 71:401-410.

67. Carrion J, Folgueira C, Alonso C: Immunization strategies against visceral leishmaniosis with the nucleosomal histones of Leishmania infantum encoded in DNA vaccine or pulsed in dendritic cells. Vaccine 2008, 26:2537-2544.

68. Ravindran R, Bhowmick S, Das A, Ali N: Comparison of BCG, MPL and cationic liposome adjuvant systems in leishmanial antigen vaccine formulations against murine visceral leishmaniasis. BMC Microbiol 2010, 10:181.

69. Mukherjee $P$, Ghosh AK, Ghose AC: Infection pattern and immune response in the spleen and liver of $B A L B / c$ mice intracardially infected with Leishmania donovani amastigotes. Immunol Lett 2003, 86:131-138.

70. Squires KE, Kirsch M, Silverstein SC, Acosta A, McElrath MJ, Murray HW: Defect in the tissue cellular immune response: experimental visceral leishmaniasis in euthymic C57BL/6 ep/ep mice. Infect Immun 1990, 58:3893-3898.

71. Rolao N, Cortes S, Gomes-Pereira S, Campino L: Leishmania infantum: mixed T-helper-1/T-helper-2 immune response in experimentally infected BALB/c mice. Exp Parasitol 2007, 115:270-276.

doi:10.1186/1297-9716-42-39

Cite this article as: Nieto et al.: Mechanisms of resistance and susceptibility to experimental visceral leishmaniosis: BALB/c mouse versus syrian hamster model. Veterinary Research 2011 42:39.

\section{Submit your next manuscript to BioMed Central and take full advantage of:}

- Convenient online submission

- Thorough peer review

- No space constraints or color figure charges

- Immediate publication on acceptance

- Inclusion in PubMed, CAS, Scopus and Google Scholar

- Research which is freely available for redistribution

Submit your manuscript a www.biomedcentral.com/submit
C Biomed Central 